Revue d'histoire de l'enfance " irrégulière »

Le Temps de l'histoire

$11 \mid 2009$

Paroles libres, paroles captives

\title{
Élise Yvorel, Les enfants de l'ombre
}

Jean-Claude Vimont

\section{(2) OpenEdition}

Journals

Édition électronique

URL : http://journals.openedition.org/rhei/3104

DOI : 10.4000/rhei.3104

ISBN : 978-2-7535-1650-2

ISSN : 1777-540X

Éditeur

Presses universitaires de Rennes

Édition imprimée

Date de publication : 1 octobre 2009

ISBN : 978-2-7535-0927-6

ISSN : 1287-2431

Référence électronique

Jean-Claude Vimont, "Élise Yvorel, Les enfants de l'ombre », Revue d'histoire de l'enfance "irrégulière " [En ligne], 11 | 2009, mis en ligne le 01 octobre 2009, consulté le 04 décembre 2020. URL : http:// journals.openedition.org/rhei/3104; DOI : https://doi.org/10.4000/rhei.3104

Ce document a été généré automatiquement le 4 décembre 2020

(c) PUR 


\title{
Élise Yvorel, Les enfants de l'ombre
}

\author{
Jean-Claude Vimont
}

1 Les enfants de l'ombre, La vie quotidienne des jeunes détenus au $\mathrm{XX}^{e}$ siècle en France métropolitaine

2 Élise Yvorel

3 Préface de Michelle Perrot

4 Rennes, PUR, 2007, 356 p.

5 ISBN : 978-2-7535-0465-3

6 L'ouvrage d'Élise Yvorel est issu de sa thèse dirigée par Frédéric Chauvaud et brillamment soutenue à l'université de Poitiers en décembre 2005. Dans la préface, Michelle Perrot insiste sur l'apport d'un travail qui comble un vide historiographique majeur : l'incarcération des mineurs de justice en prison avait été délaissée et le $\mathrm{XX}^{\mathrm{e}}$ siècle constituait un véritable angle mort de la recherche. Tous ceux qui entreprennent des études sur l'histoire de la justice en ce siècle de crises et de drames savent la difficulté du recueil de sources, inégalement versées aux dépôts d'archives publiques, disparues ou mal classées. Élise Yvorel a surmonté cette difficulté en inventoriant et en croisant les informations conservées aux Archives nationales et dans les archives des départements où étaient situés les principaux établissements. Elle offre des éclairages très précieux sur les différentes institutions spécifiques, prisons ou quartiers de mineurs, sur le temps carcéral organisé par les autorités judiciaires et enfin sur la privacy de jeunes détenu(e)s confronté(e)s à la précarité et à la violence des systèmes de correction.

7 L'introduction de l'auteur présente un panorama complet de l'état de la recherche sur la détention des mineurs, recherche principalement axée sur le $\mathrm{XIX}^{\mathrm{e}}$ siècle et sur les colonies pénitentiaires. Les quartiers de mineurs aménagés dans les prisons ont bien souvent été délaissés, à l'exception des articles sur la Petite-Roquette, des articles sur l'action 
du docteur Vingtrinier dans le quartier aménagé au sein du Bicêtre rouennais, des articles sur les quartiers correctionnels aménagés à partir de 1868 dans quelques prisons et où séjournèrent les petits Communards.

8 Dans une première partie, elle présente les quartiers d'établissements réservés aux mineurs et les processus de séparation imparfaits qui les tiennent à l'écart des adultes jusque dans les années soixante-dix. Le décalage est grand entre les principes invoqués et les pratiques de la détention. Fresnes, qui dispose d'une maison de préservation pour les filles, d'une maison d'éducation surveillée pour les garçons, puis d'un centre spécial d'observation, aurait pu sembler mieux combler les vœux des partisans d'une éducation correctionnelle adaptée à l'âge des hôtes. Élise Yvorel montre qu'il s'agit d'établissements "crypto-carcéraux » que peu de traits distinguent des maisons d'arrêt ordinaires. La prison de la Petite Roquette reçoit des mineurs jusqu'en 1928, et non jusqu'en 1865, comme il est communément admis. Dans cette prison cellulaire, on mélange toutes sortes de catégories de jeunes : orphelins, prévenus, condamnés à de courtes peines, jeunes adultes, détenus par correction paternelle. Cette situation n'est pas sans faire penser aux mélanges de catégories évoqués par Éric Pierre et Jacques Bourquin, dans les années vingt, à Mettray. Après la Libération, dans l'univers carcéral, les efforts portent sur les jeunes adultes et sur les plus jeunes des prévenus de collaboration: camps de "rééducation» installés dans d'anciens camps de concentration, dans la forteresse de Doullens, dans les prisons-écoles de l'Est. Là aussi, une certaine confusion règne. Des travaux ultérieurs seraient souhaitables pour mieux connaître les efforts de Paul Amor en direction de ces jeunes délinquants politiques. C'est avec la construction de la plus grande maison d'arrêt d'Europe que voit le jour le centre de jeunes détenus de Fleury-Mérogis, mais, là aussi, le mélange de mineurs pénaux et de mineurs civils entrave la bonne marche de l'établissement, malgré les dispositifs d'observation mis en place pour mieux cerner les personnalités. Le tableau des établissements spécifiques pour mineurs montre l'incapacité de l'Administration pénitentiaire à inventer des univers carcéraux appropriés et distincts de la prison ordinaire. Ce bilan interroge sur les initiatives de ces dernières années, pourtant présentées comme le remède ultime à la récidive.

9 La seconde partie aborde le quotidien des jeunes détenus, la manière dont leur temps est occupé, réglementé, rythmé par différentes activités. On pénètre ainsi au cœur des prisons pour suivre les jeunes au fil de jours monotones, car malgré la variété des activités imposées, l'oisiveté prédomine. L'auteur montre bien que cette dernière fut de tout temps la hantise des autorités pénitentiaires qui y voyaient la source des désordres et de la corruption des individus les uns par les autres. Les formations professionnelles et l'instruction pour tous cheminent fort lentement et recèlent bien des insuffisances, malgré l'impulsion donnée en 1945. Alimentation et soins médicaux 
s'améliorèrent au fil des années, mais les inégalités entre établissements sont flagrantes.

La troisième partie de l'ouvrage entre dans l'intimité des jeunes détenus. Cette approche des corps en prison, lavés, soignés, tondus, habillés, redressés autoritairement puis progressivement tolérés dans leur diversité est très riche. Les souffrances, privations et pratiques sexuelles clandestines sont évoquées. La manière dont les jeunes se conforment ou non à la discipline, contournent les absurdes règles du silence cellulaire longtemps en vigueur, écopent de rapports d'incidents, tentent des révoltes, (révoltes de détenus de droit commun mais aussi mouvements collectifs de jeunes collaborateurs ou de jeunes prisonniers politiques de la guerre d'Algérie), est richement illustrée d'exemples précis issus des procédures disciplinaires. Car le quotidien des quartiers de jeunes détenus est fait de différentes formes de violences atteignant plusieurs degrés de gravité. Élise Yvorel se pose bien évidemment la question des causes de la violence endémique qui a toujours été l'une des caractéristiques des établissements de mineurs, ce qu'avait déjà montré Auguste Lebreton dans Les Hauts murs,qui vient de faire l'objet d'une adaptation cinématographique. L'univers carcéral ajoute à toute peine privative de liberté de multiples souffrances, humiliations, privations, vexations qui ne peuvent que tendre les rapports entre tous les membres de ces communautés humaines. Certes, les douleurs les plus criantes ont disparu, fort lentement, mais il en demeure bien d'autres liées à la promiscuité, à la précarité née des surencombrements des prisons et à l'état de détresse psychologique des détenus.

11 Le livre d'Élise Yvorel, c'est l'envers du décor des Choristes. C'est l'évocation d'un passage obligé que les historiens avaient oublié au profit des expérimentations pénales plus ou moins généreuses. Elle montre bien quelques inflexions, quelques réformes, des initiatives innovantes et courageuses, mais ce qui prédomine est l'immobilité, la permanence des dysfonctionnements sur la longue durée. Après la lecture de ce livre, fort bien rédigé et d'une grande clarté, il faut être pourvu d'une bonne dose d'optimisme pour croire encore à l'utopie de la solution carcérale pour les mineurs... ou ne plus y croire et, comme autrefois, les abandonner à un sort comparable à celui que connurent leurs aînés. 